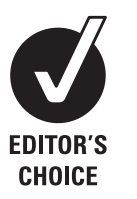

See Editorial, p2

${ }^{1}$ Brown Medical School/Miriam Hospital, Providence, Rhode Island, USA ${ }^{2}$ The Fenway Institute, Fenway Health, Boston, Massachusetts, USA ${ }^{3}$ Harvard Medical School/Massachusetts General Hospital, Boston,

Massachusetts, USA ${ }^{4}$ Harvard School of Public Health, Boston, Massachusetts, USA

\section{Correspondence to}

Dr K H Mayer, The Fenway Institute, Fenway Health, 1340 Boylston Street, Boston, MA 02215, USA;

Kenneth_Mayer@brown.edu

Accepted 10 August 2009 Published Online First 30 August 2009

\title{
Which HIV-infected men who have sex with men in care are engaging in risky sex and acquiring sexually transmitted infections: findings from a Boston community health centre
}

\author{
K H Mayer, ${ }^{1}$ C O'Cleirigh, ${ }^{2,3}$ M Skeer, ${ }^{2,4}$ C Covahey, ${ }^{2}$ E Leidolf, ${ }^{2}$ R Vanderwarker, ${ }^{2}$ \\ S A Safren ${ }^{2,3}$
}

\begin{abstract}
Objectives The primary objective was to determine the prevalence of sexually transmitted infections (STI) in a cohort of HIV-infected men who have sex with men (MSM) in their primary care setting, and to identify the demographic and behavioural characteristics of those infected with STI and the correlates of sexual transmission risk behaviour.
\end{abstract}

Methods At study entry, participants ( $n=398$ ) were tested for STI and their medical charts were reviewed for STI results in the previous year. Data on demographics, substance use, sexual behaviour and HIV disease characteristics were collected through a computerassisted self-assessment and medical record extraction. Logistic regression analyses assessed characteristics of those with recent STI and recent transmission risk behaviour.

Results The sample was predominantly white (74.6\%) and college educated (51.7\%). On average, participants were 41.5 years old (SD 8.4) and had been HIV infected for 8.6 years (SD 6.7); $9 \%$ of the sample had an STI, with $6.4 \%$ testing positive for syphilis, 3.1\% for gonorrhoea and $0.25 \%$ for chlamydia. Age and years since HIV diagnosis were significantly associated with testing positive for an STI, as was engaging in transmission risk behaviour and using methamphetamine, ketamine and inhalants. Substance use, particularly methamphetamine use, and being more recently diagnosed with HIV were each uniquely associated with transmission risk behaviour in a multivariable model.

Conclusions These results underscore the need to develop more effective secondary prevention interventions for HIV-infected MSM, tailored to more recently diagnosed patients, particularly those who are younger and substance users.

The Centers for Disease Control and Prevention has documented a dramatic increase in the rates of sexually transmitted diseases among men who have sex with men (MSM) as evidenced by reports from major urban centres across the country over the past 10 years. ${ }^{1-5}$ In addition, the HIV incidence among MSM has been increasing consistently since the early 1990s, and MSM currently represent the group with the largest number of new HIV infections $(53 \%)$ in the USA. ${ }^{6}$ Based upon national surveillance at sexually transmitted disease clinics, median positivity rates for gonorrhoea and chlamydia are higher among HIV-infected MSM than among HIV-uninfected or unknown status MSM. Syphilis seroreactivity rates are up to four times greater among HIV-infected MSM than HIV-uninfected or unknown status MSM. ${ }^{7}$ This trend of higher comparative rates of incident sexually transmitted infection (STI) among HIV-infected MSM is also evident in community samples both nationally ${ }^{8}$ and internationally. ${ }^{9-11}$

Consistent with high rates of STI among HIVinfected MSM, some studies suggest that rates of high-risk sexual behaviours (ie, anal or vaginal intercourse without a condom) may be up to three times higher among HIV-infected MSM compared with HIV-uninfected MSM $^{12} 13$ and higher than HIV-infected men who have sex with women. ${ }^{14}$ Among community-recruited samples of HIVinfected MSM, unprotected anal intercourse has been significantly associated with substance use including methamphetamines and erectile dysfunction medications. ${ }^{12} 1415$ In addition, sexual transmission risk behaviour (ie, serodiscordant unprotected anal intercourse) in MSM has been significantly related to substance use (including methamphetamines), non-disclosure of serostatus to all sexual partners and low coping self-efficacy. ${ }^{16}$ Among those in clinical care, transmission risk behaviour has been associated with younger age, daily alcohol use and not currently taking antiretroviral therapy. ${ }^{17}$

The presence of high rates of STI among HIVinfected MSM and continuing high rates of sexual risk behaviour with serodiscordant sexual partners ${ }^{18}$ presents an increased risk for the sexual health of all MSM with HIV, and raises public health concerns for the increased risk of HIV transmission. The current study supports the published research by providing more recent prevalence data on STI among an urban sample of HIVinfected MSM living in the northeast. This study extends the existing research by relating particular demographic, disease and health risk behavioural characteristics to STI likelihood. Therefore, the purpose of this analysis is to: (1) report on the prevalence of gonorrhoea, chlamydia and syphilis among a cohort of HIV-infected MSM screened for participation in an HIV prevention intervention who are receiving primary care at Fenway Health; (2) report on the rates of sexual transmission risk behaviour among this group; and (3) identify the significant correlates of STI and sexual transmission risk behaviour. 


\section{METHODS}

\section{Sample and data collection}

Sample

Participants were 398 HIV-infected MSM screened for participation in an HIV prevention intervention trial through Fenway Health, the largest ambulatory facility caring for MSM in New England, and tested for STI at study entry, or a chart review indicated that they had received STI testing in the year before study assessment. Men who met the inclusion criteria for the prevention intervention trial were: (1) infected with HIV; (2) received primary HIV care at Fenway Health; (3) were 18 years of age or older; and (4) self-identified as MSM. This study utilised cross-sectional, baseline measures from the prevention intervention trial screening.

\section{Collection of biological markers}

Participant recruitment for the 12-month study began in July 2004 and participant follow-up continued to August 2008. To assess diagnoses of STI, participants were tested for syphilis, gonorrhoea and chlamydia. Syphilis screening of blood samples utilised the rapid plasma reagin method and reactive serologies had confirmatory fluorescent treponemal antibody absorption performed. Specimen collection to detect gonococcal and chlamydia urethritis was performed using urine samples via nucleic acid amplification testing. The prevalence of rectal and pharyngeal gonorrhoea and chlamydia was extracted from the participants' medical records. Testing methodology before August 2006 utilised PCR technology. Subsequent to August 2006, the amplification method used was changed to strand displacement amplification, as a result of changes in available assays at the testing laboratory (Quest).

CD4 cell count and plasma HIV-RNA were routinely measured at least quarterly as part of ongoing primary care. With permission from the participants, the results of these biological measures were captured from the lab server (Quest) or via hard copy report, and translated or entered into an electronic medical record system (Logician/Centricity EMR), followed by extraction into a Microsoft Access database.

\section{Collection of behavioural data}

Behavioural data were collected via surveys administered through the audio computer-assisted self-interview system that were given during the screen-in process for participation in the prevention intervention study. The survey lasted for approximately one hour and included the measures below.

\section{Measures}

The primary dependent variable was a dichotomous indicator of having been diagnosed with a bacterial STI (syphilis, gonorrhoea or chlamydia) within the past year. The primary independent variable was transmission risk behaviour, operationalised as whether or not participants had unprotected insertive or receptive anal sex with possible serodiscordant (HIV-negative or unknown status) partners within the past 6 months. Furthermore, we examined the unprotected insertive and receptive anal sex components of transmission risk behaviour as separate independent variables. Additional independent variables of interest included demographics (age, race/ethnicity, education and annual income), HIV disease status and treatment (number of years since being diagnosed with HIV, CD4 cell count, undetectable viral load (less than 75 copes $/ \mathrm{ml}$ ) and currently being on antiretroviral medications), mental health indicators (posttraumatic stress disorder symptoms, depression and anxiety) and past 3-month dichotomous substance use indicators (binge drinking (ie, consuming five or more alcoholic beverages in a single day at least once), any crystal methamphetamine use, any use of other specific drugs (ie, marijuana, crack, cocaine, heroin, ketamine, opiates, tranquilizers or barbiturates, hallucinogens, or inhalants) and a composite of other drug use).

Mental health indicators were assessed through various psychosocial batteries in the audio computer-assisted self-interview. Depression was defined as screening positive for either major depressive syndrome or other depressive syndrome within the past 2 weeks, as measured by the nine-item depression severity scale of the patient health questionnaire (PHQ). ${ }^{19}$ In addition, patients were considered to have an anxiety disorder if they met screen-in criteria for any of the following disorders: panic disorder, as measured by the panic disorder severity scale of the $\mathrm{PHQ},{ }^{19}$ other anxious syndrome, as assessed by the $\mathrm{PHO},{ }^{19}$ social phobia, as captured by the MINI-SPAN, ${ }^{20}$ and posttraumatic stress disorder, as measured by the four-item SPAN. ${ }^{21}$

\section{Data analysis}

Analyses were conducted using SPSS and SAS statistical software packages. Descriptive statistics were assessed for all variables. Bivariate logistic regression analyses were conducted to assess the relationship between the outcome (STI) and each independent variable. Because most of the variation in STI can be accounted for by transmission risk behaviour (as the predominant method for contracting syphilis, gonorrhoea and chlamydia among MSM is through unprotected anal intercourse), we conducted a second set of bivariate logistic regression models examining the effect of the independent variables (excluding sexual risk behaviour) on the dichotomous transmission risk behaviour outcome. Due to the overlap in variation with the independent variables and the outcomes, and the redundancy of running multivariable models with both outcomes (STI and transmission risk behaviour), we a priori chose only to present the multivariable model of the intervening outcome (transmission risk behaviour). As such, variables that were statistically significant $(p<0.05)$ in the second set of bivariate analyses were retained in a multivariable logistic regression analysis, which controlled for race/ethnicity and education, regardless of significance level. Furthermore, in the case in which two significant variables were highly correlated, we chose to include only one in the final model to eliminate the threat of multicollinearity posed by putting highly correlated variables in the same multivariable model. In that regard, although multiple drugs were examined individually in the bivariate models, in the multivariable model, we only included a dichotomous composite measure of any drug use (excluding methamphetamine) and crystal methamphetamine use.

\section{RESULTS}

\section{Sample characteristics}

The sample characteristics are presented in table 1

Less than $10 \%$ of the sample had been diagnosed with an STI within the past year, and among those who had $(n=36), 69.4 \%$ had been diagnosed with syphilis, $33.3 \%$ with gonorrhoea and $2.8 \%$ with chlamydia. Of the gonorrhoea and chlamydia diagnoses $(n=13), 61.5 \%$ were from a urethral source, $30.8 \%$ were rectal and $7.7 \%$ were pharyngeal. Of the nine participants whose STI was diagnosed as part of their primary care, five were tested in response to clinical symptom presentation and four of those tested were asymptomatic but reported possible exposure.

The mean age of the participants was 41.5 years (SD 8.4), $43.3 \%$ of the participants had an annual income greater than US 
Table 1 Profile of the sample $(n=398)$

\begin{tabular}{|c|c|}
\hline Characteristic & Percentage or mean (SD) \\
\hline \multicolumn{2}{|l|}{ Sexual risk } \\
\hline $\begin{array}{l}\text { Had been diagnosed with an STI (past } \\
\text { year) }\end{array}$ & $9.0 \%$ \\
\hline \multicolumn{2}{|l|}{$\begin{array}{l}\text { Type of STI (among those with an STI } \\
\text { diagnosis) }\end{array}$} \\
\hline Syphilis (389 tested) & $6.3 \%$ \\
\hline Gonorrhoea (228 tested) & $5.3 \%$ \\
\hline Chlamydia (232 tested) & $0.4 \%$ \\
\hline $\begin{array}{l}\text { Transmission risk behaviour (past } 6 \\
\text { months) }\end{array}$ & $50.5 \%$ \\
\hline \multicolumn{2}{|l|}{ Demographics } \\
\hline Age, years & 41.5 (8.4); range $20-68$ \\
\hline \multicolumn{2}{|l|}{ Race/ethnicity } \\
\hline White & $74.6 \%$ \\
\hline Black/African American & $10.8 \%$ \\
\hline Hispanic/Latino & $9.8 \%$ \\
\hline Other & $4.8 \%$ \\
\hline \multicolumn{2}{|l|}{ Education } \\
\hline High school degree/GED & $13.6 \%$ \\
\hline $\begin{array}{l}\text { Some college/associates degree/ } \\
\text { technical degree }\end{array}$ & $34.7 \%$ \\
\hline College degree & $34.7 \%$ \\
\hline Graduate degree & $17.0 \%$ \\
\hline \multicolumn{2}{|l|}{ Annual income } \\
\hline Less than US\$20 000 & $35.1 \%$ \\
\hline US\$20 001-40 000 & $21.6 \%$ \\
\hline US\$40 001-60 000 & $15.3 \%$ \\
\hline Greater than US\$60 000 & $28.0 \%$ \\
\hline \multicolumn{2}{|l|}{ Past 3 months substance use } \\
\hline $\begin{array}{l}\text { Binge drinking (5 or more drinks in one } \\
\text { sitting) }\end{array}$ & $19.3 \%$ \\
\hline Crystal methamphetamine use (any) & $22.9 \%$ \\
\hline Other drug use (any) & $56.8 \%$ \\
\hline \multicolumn{2}{|l|}{ HIV disease status and treatment } \\
\hline Years since HIV diagnosis & $8.6(6.7)$; range $0.2-22.4$ \\
\hline CD4 cell count (cells $/ \mathrm{mm}^{3}$ ) & 532.1 (299.3); range $14-1987$ \\
\hline Currently taking HIV medication & $66.1 \%$ \\
\hline $\begin{array}{l}\text { Viral load (mean plasma HIV-RNA, } \\
\text { copies/ml) }\end{array}$ & 14777 (46 332); range 75-442 298 \\
\hline
\end{tabular}

$\$ 40000$, and the sample was largely white (74.6\%) and college educated $(51.7 \%)$. On average, participants had been diagnosed with HIV for 8.6 years (SD 6.7), had a CD4 cell count of 523.1 cells $/ \mathrm{mm}^{3}$ (SD 299.3) and had a mean plasma HIV-RNA of 14 777 copies/ml (SD 46332 ). Approximately half (54.5\%) of the sample had an undetectable viral load and $66.1 \%$ were taking anti-HIV medication at the time of the survey. Within the 3 months before the survey, $19.3 \%$ of the sample reported binge drinking, $22.9 \%$ reported using crystal methamphetamine and $56.8 \%$ reported using other drugs. Approximately half (50.5\%) of the sample reported engaging in transmission risk behaviour within the past 6 months.

\section{Logistic regression analyses}

The bivariate logistic regression analyses that examined the effect of the independent variables on STI demonstrated that of all of the demographic, sexual risk, HIV, mental health and substance use indicators, the following variables were statistically significantly associated with having been diagnosed with an STI within the past year: transmission risk behaviour (odds ratio (OR) 4.41, 95\% CI 1.88 to 10.36 ); unprotected serodiscordant insertive anal sex (OR 2.11, 95\% CI 1.04 to 4.30 ); unprotected serodiscordant receptive anal sex (OR 3.86, 95\% CI
1.78 to 8.28 ); age (standardised) (OR $0.63,95 \%$ CI 0.44 to 0.91 ); number of years since HIV diagnosis (standardised) (OR 0.66 , 95\% CI 0.45 to 0.97 ); and crystal methamphetamine (OR 3.37, $95 \%$ CI 1.67 to 6.81 ), ketamine (OR $4.48,95 \%$ CI 1.83 to 11.00$)$ and inhalant use (OR 2.60, 95\% CI 1.28 to 5.30 ).

Similarly, in the additional bivariate analyses, age (OR 0.76 , $95 \%$ CI 0.62 to 0.94 ), number of years since HIV diagnosis (OR $0.57,95 \%$ CI 0.46 to 0.70 ), crystal methamphetamine use (OR $4.25,95 \%$ CI 2.45 to 7.38 ), ketamine use (OR 7.66, 95\% CI 2.62 to 22.34 ) and inhalant use (OR 4.22, 95\% CI 2.62 to 6.78) were all significantly associated with transmission risk behaviour at baseline (table 2). Additional significant independent variables included: use of marijuana (OR 1.86, 95\% CI 1.21 to 2.84), tranquilizers or barbiturates (OR 2.00, 95\% CI 1.03 to 3.89 ), hallucinogens (OR 4.07, 95\% CI 1.81 to 9.16), viagra (OR 3.64, $95 \%$ CI 2.09 to 6.36); a composite measure of other drug use (OR $3.18,95 \%$ CI 2.08 to 4.85 ) and having a detectable viral load (OR $1.68,95 \%$ CI 1.12 to 2.53 ).

Due to the high correlation between age and years since HIV diagnosis $(r=0.44 ; p<0.001)$, only the number of years since HIV diagnosis was included in the multivariable model, as the focus of this study was the HIV-related correlates of transmission risk behaviour. In the final multivariable model, years since HIV 
Table 2 Results from the bivariate and multivariable logistic regression analyses with the outcome of transmission risk behaviour

\begin{tabular}{|c|c|c|c|c|}
\hline & $\begin{array}{l}\text { Unadjusted OR } \\
(95 \% \text { CI })\end{array}$ & $\begin{array}{l}\mathbf{p} \\
\text { Value }\end{array}$ & $\begin{array}{l}\text { Adjusted OR* } \\
(95 \% \mathrm{CI})\end{array}$ & $\begin{array}{l}\mathbf{p} \\
\text { Value }\end{array}$ \\
\hline Age (standardised) & $0.76(0.62$ to 0.94$)$ & 0.011 & NA & \\
\hline Years since HIV diagnosis (standardised) & $0.57(0.46$ to 0.70$)$ & $<0.001$ & $0.60(0.48$ to 0.76$)$ & $<0.001$ \\
\hline \multicolumn{5}{|l|}{ Crystal methamphetamine use } \\
\hline No & 1.00 & & 1.00 & \\
\hline Yes & 4.25 (2.45 to 7.38$)$ & $<0.001$ & $2.57(1.37$ to 4.82$)$ & 0.003 \\
\hline \multicolumn{5}{|l|}{ Other drug use } \\
\hline No & 1.00 & & 1.00 & \\
\hline Yes & $3.18(2.08$ to 4.85$)$ & $<0.001$ & 2.17 (1.32 to 3.58$)$ & 0.002 \\
\hline \multicolumn{5}{|l|}{ Viral load } \\
\hline$\leq 75$ & 1.00 & & 1.00 & \\
\hline$>75$ & $1.68(1.12$ to 2.53$)$ & 0.012 & $1.14(0.72$ to 1.80$)$ & 0.586 \\
\hline
\end{tabular}

diagnosis, drug use and crystal methamphetamine use all remained significant (table 2 ). These results indicated that each additional year since HIV diagnosis was associated with a reduced odds (OR $0.60,95 \%$ CI 0.48 to 0.76 ) of transmission risk behaviour, and that men who reported using crystal methamphetamine within the past 3 months (OR 2.57, 95\% CI 1.37 to 4.82 ) or men who reported using any recreational drugs within the past 3 months (OR 2.17, 95\% CI 1.32 to 3.58 ) had a greater odds of transmission risk behaviour. Having a detectable viral load was no longer statistically significant in the multivariable model, which was due, at least partly, to the significant correlations $(p<0.01)$ between this variable and other variables in the model, including crystal methamphetamine use and the number of years since HIV diagnosis.

\section{DISCUSSION}

The results of this study indicate that $9.0 \%$ of HIV-infected MSM in care in a Boston community health centre had been diagnosed with at least one STI during a one-year period, with reactive syphilis tests accounting the majority of cases. The presence of STI can compromise the health of people living with HIV through several mechanisms, including increasing susceptibility to reinfection and superinfection by disrupting mucosal barriers to infection or by increasing the presence of HIVsusceptible inflammatory cells in the genital tract. ${ }^{22}$ In addition, several recent reports have documented significant acute decreases in CD4 cell counts and acute increases in HIV viral RNA in response to syphilis infection, ${ }^{23-25}$ which could compromise effective antiretroviral therapy. Moreover, the presence of STI among HIV-infected MSM already in care provides convincing evidence of ongoing risky sexual behaviour in this group, creating risks for HIV transmission to uninfected sexual partners, because STI may increase HIV infectiousness by facilitating HIV shedding in the genital tract or the rectal mucosa. ${ }^{22}$

The results of the current study also identified that HIVinfected MSM who were younger or more recently diagnosed with HIV, or who reported methamphetamine, ketamine, or inhalant use in the past 3 months or unprotected anal intercourse in the past 6 months, were significantly more likely to have had a new bacterial STI result in the past year. Slightly more than half the sample reported one or more episode of unprotected anal intercourse with a serodiscordant partner in the past 6 months, tended to be more recently diagnosed with $\mathrm{HIV}$, and were more likely to have used methamphetamine or other recreational drugs in the previous 3 months. These results from this Boston cohort are consistent with the characteristics of HIV-infected MSM who engage in transmission risk behav- iour in other metropolitan centres throughout the USA (ie, Chicago, Los Angeles, New York, San Francisco). ${ }^{12}$ 14-16 26

The increased incidence of syphilis in this cohort may be reflective of serosorting, when men may selectively disclose their HIV status and/or have unprotected sex with more HIV-infected partners in order to be able to have unprotected anal sex without the concern of transmitting to others, which could concentrate the syphilis epidemic among HIV-infected MSM. The increased incidence of syphilis in the current sample may also represent the fact that HIV-infected MSM may be more susceptible to contracting syphilis compared with their uninfected counterparts. ${ }^{27}$ Furthermore, a recent review of secular trends of STI at Fenway Health over the past decade documents that syphilis has been increasing, revealing that approximately half of the new cases of syphilis at the health centre are among HIV-infected MSM, even though these men represent less than $20 \%$ of the full MSM population getting primary care at Fenway Health. ${ }^{28}$

These results are most appropriately interpreted with a consideration of the limitations of this study. These analyses are presented within a cross-sectional design and as such neither causal relationships nor the directionality of the relationship can be inferred. Several of the measures were based upon patient self-report and are vulnerable to the biases of that methodology. In addition, information concerning the prevalence of STI reported here was obtained from both study-related procedures at study entry and through STI assays conducted through clinical care, which may contribute to bias in estimating stable STI rates in this sample. Finally, the sample only included men who received primary care, specifically at Fenway Health, and was mostly white $(75 \%)$, which limits the generalisability of the findings. However, because Massachusetts provides universal access to health care and there is state support to supplement Ryan White (federal aid) programmes, there is no reason to expect that HIV-infected MSM clients receiving care at Fenway Health would differ radically from other HIV-infected MSM in care in other HIV specialty clinics in the Boston area. However, as Fenway Health is known for providing comprehensive services to the region's gay and lesbian communities, it is possible that some potential clients who want less public awareness of their sexual orientation and/or HIV status could seek care elsewhere, so that the cohort in this study might be riskier than men receiving care at other centres, but rigorous comparative data are not available. Participants in this study had to have an established relationship with the healthcare centre and sign an informed consent, so some individuals with more serious mental health concerns and/or unstable housing, who might also be riskier sexually, might not be included in this sample. 


\section{Key messages}

- STI rates among HIV-infected MSM are high, as are rates of risky sexual behaviours.

- This study demonstrates that younger age, fewer years since HIV diagnosis and drug use are associated with developing incident STI, and with engaging in transmission risk behaviour.

- Innovative secondary prevention interventions for HIV-infected MSM, particularly those who are younger and substance users, may help to decrease risk taking in this subpopulation.

The high rates of HIV and STI transmission risk behaviour reported among HIV-infected Boston MSM suggest that the development of effective secondary HIV prevention intervention programmes should be a public health priority. These results also suggest that these programmes could be maximally effective if they focus on younger or more recently diagnosed MSM, who may not have had sufficient provider contact to receive intensive prevention counselling. Given the high rate of co-infectiousness among these HIV-infected MSM, it may be particularly important for prevention efforts to be integrated into primary care as a key syndemic approach to improving the sexual health of young, recently diagnosed HIV-positive MSM or those with drug use issues. Furthermore, the high prevalence of co-infections in this population may have implications for biomedical approaches to HIV prevention, including incorporating preexposure prophylaxis and "test and treat" strategies in prevention efforts. Further longitudinal research with biomedical approaches would be well suited to examine these relationships further.

Innovative programmes that facilitate education and skills building around safer sex when MSM are relatively recently diagnosed and entering care may help to decrease risk taking in this subpopulation. Effective care programmes may also enhance HIV prevention by providing substance use screening, treatment and/or referral options for HIV-infected MSM in care. Similarly, effective prevention for positive programmes and access to treatment for concomitant STI will address the dual priorities of reducing transmission of HIV and other STI and will improve the sexual health and HIV disease management among those living with HIV.

Contributors KHM is the principal investigator of the study, conceptualised the manuscript and led the manuscript writing. CO and MS conducted the statistical analyses and contributed to the preparation of the manuscript. CC, EL and RV consulted on the project and contributed to the preparation of the manuscript. SAS is the co-principal investigator of the study and contributed to the development of the assessments used in the study and the preparation of the manuscript.

Funding This study was supported by NIMH grant 5R01MH068746-05 and HRSA grant H97HA01293 awarded to KHM and SAS.

Competing interests None.

Ethics approval Ethics approval was obtained from Fenway Health, Boston, MA.

Patient consent Obtained.

Provenance and peer review Not commissioned; externally peer reviewed.

\section{REFERENCES}

1. Centers for Disease Control and Prevention. Trends in primary and secondary syphilis and HIV infections in men who have sex with men-San Francisco and Los Angeles, California, 1998-2002. MMWR Morb Mortal Wkly Rep 2004;53:575-8.
2. Centers for Disease Control and Prevention. Primary and secondary syphilis among men who have sex with men-New York City, 2001. MMWR Morb Mortal Wkly Rep 2002;51:853-6.

3. Centers for Disease Control and Prevention. Outbreak of syphilis among men who have sex with men-Southern California, 2000. MMWR Morb Mortal Wkly Rep 2001:50:117-20.

4. Centers for Disease Control and Prevention. Primary and secondary syphilis-United States, 2003-2004. MMWR Morb Mortal Wkly Rep 2006:55:269-73.

5. Heffelfinger JD, Swint EB, Berman SM, et al. Trends in primary and secondary syphilis among men who have sex with men in the United States. Am J Public Health 2007:97:1076-108.

6. Centers for Disease Control and Prevention (CDC). Fact sheet: estimates of new HIV infections in the United States, August 2008. http://www.cdc.gov/hiv/topics/ surveillance/resources/factsheets/pdf/incidence.pdf (accessed 7 July 2009).

7. Centers for Disease Control and Prevention (CDC). Men who have sex with men-STD surveillance 2006. http://www.cdc.gov/STD/stats06/msm.htm laccessed 21 May 2008)

8. Whittington WL, Collis T, Dithmer-Schreck D, et al. Sexually transmitted diseases and human immunodeficiency virus-discordant partnerships among men who have sex with men. Clin Infect Dis 2002;35:1010-17.

9. Dodds JP. A tale of three cities: persisting high HIV prevalence, risk behaviour and undiagnosed infection in community samples of men who have sex with men. Sex Transm Infect 2007;83:392-6.

10. Fengyi J. High rates of sexually transmitted infections in HIV positive homosexual men: data from two community based cohorts. Sex Transm Infect 2007;83:397-9.

11. Dougan S, Evans BG, Elford J. Sexually transmitted infections in Western Europe among HIV-positive men who have sex with men. Sex Transm Dis 2007:34:783-90.

12. Carey JW, Mejia R, Bingham T, et al. Drug use, high risk sexual behavior, and increased risk for recent HIV infection among men who have sex with men in Chicago and Los Angeles. AIDS Behav. Published Online First: 23 May 2008. doi:10.1007/ S10461-008-9403-3

13. Brewer DD, Golden MR, Handsfield HH. Unsafe sexual behavior and correlates of risk in a probablility sample of men who have sex with men in the era of highly active antiretroviral therapy. Sex Transm Dis 2006;33:250-5.

14. Morin SF, Myers JJ, Shade SB, et al. Predicting HIV transmission risk among HIVinfected patients seen in clinical settings. AIDS Behav 2007;11(5 Suppl):6-16.

15. Spindler HS, Scheer S, Chen SY, et al. Viagra, methamphetamine, and HIV risk: results from a probability sample of MSM, SanFrancisco. Sex Transm Dis 2007:34:1-6.

16. Morin SF, Steward WT, Charlebois ED, et al. Predicting HIV transmission risk among HIV-infected men who have sex with men: findings from the healthy living project. $J$ Acquir Immune Defic Syndr 2005;40:226-35

17. Drumright LN, Little SJ, Strathdee SA, et al. Unprotected anal intercourse and substance use among men who have sex with men with recent HIV infection. J Acquir Immune Defic Syndr 2006;43:344-50.

18. Fenton KA, Imrie J. Increasing rates of sexually transmitted diseases in homosexual men in Western Europe and the United States: why? Infect Dis Clin North Am 2005; 19:311-31

19. Spitzer RL, Korenke K, Williams JBW, for the Patient Health Questionnaire Primary Care Study Group. Validation and utility of a self-report version of PRIME-MD: the PHO Primary Care Study. JAMA 1999;282:1737-44.

20. Connor KM, Kobak KA, Churchill LE, et al. Mini-SPIN: a brief screening assessment for generalized social anxiety disorder. Depress Anxiety 2001;14:137-40.

21. Meltzer-Brody S, Churchill E, Davidson JR. Derivation of the SPAN, a brief diagnostic screening test for post-traumatic stress disorder. Psychiatry Res 1999;88:63-70.

22. Fleming DT, Wasserheit JN. From epidemiological synergy to public health policy and practice: the contribution of other sexually transmitted diseases to sexual transmission of HIV infection. Sex Transm Infect 1999;75:3-17.

23. Palacios R, Jiménez-Oñate $F$, Aguilar M, et al. Impact of syphilis infection on HIV viral load and CD4 cell counts in HIV-infected patients. J Acquir Immune Defic Syndr 2007:44:356-9.

24. Buchacz K, Patel P, Taylor M, et al. Syphilis increases HIV viral load and decreases CD4 cell counts in HIV-infected patients with new syphilis infections. AIDS 2004; 18:2075-9

25. Kofoed K, Gerstoft J, Mathiesen LR, et al. Syphilis and human immunodeficiency virus (HIV)-1 coinfection: influence on CD4 T-cell count, HIV-1 viral load, and treatment response. Sex Transm Dis 2006;33:143-8.

26. Ostrow DG, Plankey MW, Cox C, et al. Specific sex drug combinations contribute to the majority of recent HIV seroconversions among MSM in the MACS. J Acquir Immune Defic Syndr 2009;51:349-55.

27. Cohen MS. When people with HIV get syphilis: triple jeopardy. Sex Transm Dis 2006;33:149-50.

28. Mimiaga MJ, Helms DJ, Reisner SL, et al. Gonococcal, chlamydia, and syphilis infection positivity among MSM attending a large primary care clinic, Boston, 2003 to 2004. Sex Transm Dis 2009;36:507. 